\title{
ON SOME HYPERSURFACES AND HOLOMORPHIC MAPPINGS
}

\author{
Dedicated to Professor Hirotaka Fujimoto on his sixtieth birthday
}

\author{
MANABU SHIROSAKI
}

\section{§1. Introduction}

In [S], the author gave a homogeneous polynomial $H_{n}$ of variables $w_{0}, \ldots, w_{n}$ with the property:

If two algebraically non-degenerate holomorphic mappings $f$ and $g$ of $C$ into $\boldsymbol{P}^{n}(\boldsymbol{C})$ with reduced representations $\tilde{f}$ and $\tilde{g}$ respectively satisfy $H_{n}(\tilde{g})=\alpha H_{n}(\tilde{f})$ for some entrie function $\alpha$ without zero, then $f=g$.

From this, we can get a hypersurface in the complex projective space with the property that two algebraically non-degenerate holomorphic mappings of $C$ into the complex projective space which have the same inverse images as divisors are identical.

In this paper, we give another polynomial different from the above one with this property and others.

Acknowledgements. The author would like to thank the referee for valuable suggestions.

\section{§2. Previous results}

We use the terminology in [S]. In this section we recall the results in [S].

Let $f$ be a holomorphic mapping of $\boldsymbol{C}$ into $\boldsymbol{P}^{n}(\boldsymbol{C})$ with a representation $\tilde{f}=\left(f_{0}, \ldots, f_{n}\right)$. If $f(z)=\left(c_{0}: \cdots: c_{n}\right)$ for all $z \in C-\tilde{f}^{-1}(\mathbf{o})$, where $c_{0}, \ldots, c_{n}$ are constants at least one of which are not 0 , then we say that $f$ or $\left(f_{0}: \cdots: f_{n}\right)$ is constant and write $f=\left(f_{0}: \cdots: f_{n}\right)=\left(c_{0}: \cdots: c_{n}\right)$.

We will need the following:

THEOREM A ([S, p. 291]). Let $f$ be a nonconstant holomorphic mapping of $\boldsymbol{C}$ into $\boldsymbol{P}^{1}(\boldsymbol{C})$ with a reduced representation $\left(f_{0}, f_{1}\right)$ and $\left\{\left(w_{0}: w_{1}\right) \in \boldsymbol{P}^{1}(\boldsymbol{C})\right.$ : $\left.a_{j 0} w_{0}+a_{j 1} w_{1}=0\right\}(1 \leq j \leq q)$ distinct hyperplanes in $\boldsymbol{P}^{1}(\boldsymbol{C})$. If all the zeros of $a_{0 j} f_{0}+a_{j 1} f_{1}$ have the multiplicities at least $m_{j}$ for each $j$, where $m_{j}$ are arbitrarily

Received July 11, 1997; revised November 19, 1997. 
fixed positive integers $(1 \leq j \leq q)$, then

$$
\sum_{j=1}^{q}\left(1-\frac{1}{m_{j}}\right) \leq 2
$$

Let $d$ and $p$ be two positive integers with $d>2 p+8$ and $p>2$ which have no common factors. Define homogeneous polynomials $H_{1}\left(w_{0}, w_{1}\right)=$ $w_{0}^{d}+w_{0}^{p} w_{1}^{d-p}+w_{1}^{d}$ with degree $d$ and

$$
H_{n}\left(w_{0}, \ldots, w_{n}\right)=H_{1}\left(H_{n-1}\left(w_{0}, \ldots, w_{n-1}\right), w_{0}^{d^{n-1}-1} w_{n}\right)
$$

with degree $d^{n}$ for $n \geq 2$.

THEOREM B ([S, p. 297]). Let $f$ and $g$ be algebraically non-degenerate holomorphic mappings of $\boldsymbol{C}$ into $\boldsymbol{P}^{n}(\boldsymbol{C})$ with representations $\tilde{f}=\left(f_{0}, \ldots, f_{n}\right)$ and $\tilde{g}=\left(g_{0}, \ldots, g_{n}\right)$ respectively. If

$$
H_{n}\left(g_{0}, \ldots, g_{n}\right)=\alpha H_{n}\left(f_{0}, \ldots, f_{n}\right)
$$

holds for some entire function $\alpha$ without zero, then

$$
g_{j}=\beta f_{j} \quad(0 \leq j \leq n),
$$

where $\beta$ is an entire function such that $\beta^{d^{n}}=\alpha$.

LEMMA C ([S, p. 295]). Let $f$ and $g$ be algebraically non-degenerate holomorphic mappings of $\boldsymbol{C}$ into $\boldsymbol{P}^{1}(\boldsymbol{C})$ with reduced representations $\tilde{f}=\left(f_{0}, f_{1}\right)$ and $\tilde{g}=\left(g_{0}, g_{1}\right)$ respectively. If

$$
H_{1}\left(g_{0}, g_{1}\right)=h^{d} H_{1}\left(f_{0}, f_{1}\right)
$$

holds for some meromorphic function $h$, then $h$ is an entire function without zero.

Remark. For holomorphic mappings into $\boldsymbol{P}^{1}(\boldsymbol{C})$, algebraic non-degeneracy coincides with nonconstantness.

We give a new version of the case of $n=1$ of Theorem $\mathrm{B}$.

THEOREM 2.1. Let $f$ and $g$ be algebraically non-degenerate holomorphic mappings of $\boldsymbol{C}$ into $\boldsymbol{P}^{1}(\boldsymbol{C})$ with representations $\tilde{f}=\left(f_{0}, f_{1}\right)$ and $\tilde{g}=\left(g_{0}, g_{1}\right)$ respectively. If

$$
H_{1}\left(g_{0}, g_{1}\right)=h^{d} H_{1}\left(f_{0}, f_{1}\right)
$$

holds for some meromorphic function $h \neq \equiv$, then

$$
g_{j}=\varphi f_{j} \quad(j=0,1),
$$

where $\varphi$ is a meromorphic function such that $\varphi^{d}=h^{d}$. 
Proof. Let $A$ be a common factor of $f_{0}$ and $f_{1}$, and let $B$ be a common factor of $g_{0}$ and $g_{1}$. Then

$$
B^{d} H_{1}\left(\frac{g_{0}}{B}, \frac{q_{1}}{B}\right)=h^{d} A^{d} H_{1}\left(\frac{f_{0}}{A}, \frac{f_{1}}{A}\right)
$$

By Lemma $\mathrm{C},(A / B) h$ is an entire function without zeros. Hence, we get, by Theorem B,

$$
g_{j} / B=\beta f_{j} / A \quad(j=0,1),
$$

where $\beta$ is an entire function such that $\beta^{d}=((A / B) h)^{d}$. If we put $\varphi=(B / A) \beta$, then we have the conclusion. Q.E.D.

We give other homogeneous polynomials different from the above $H_{n}$. Let $P\left(w_{0}, w_{1}\right)=P_{1}\left(w_{0}, w_{1}\right)=H_{1}\left(w_{0}, w_{1}\right)$ and define inductively

$$
P_{n}\left(w_{0}, \ldots, w_{n}\right)=P_{n-1}\left(P\left(w_{0}, w_{1}\right), \ldots, P\left(w_{n-1}, w_{n}\right)\right)
$$

with degree $d^{n}$ for $n \geq 2$. In place of $H_{n}$, we consider $P_{n}$ in this paper.

\section{§3. Uniqueness of holomorphic mappings}

First, we prove the following uniqueness theorem:

THEOREM 3.1. Let $f$ and $g$ be algebraically non-degenerate holomorphic mappings of $\boldsymbol{C}$ into $\boldsymbol{P}^{n}(\boldsymbol{C})$ with representations $\tilde{f}=\left(f_{0}, \ldots, f_{n}\right)$ and $\tilde{g}=\left(g_{0}, \ldots, g_{n}\right)$ respectively. If

$$
P_{n}\left(g_{0}, \ldots, g_{n}\right)=h^{d^{n}} P_{n}\left(f_{0}, \ldots, f_{n}\right)
$$

holds for some meromorphic function $h \not \equiv 0$, then

$$
g_{j}=\omega_{n} h f_{j} \quad(0 \leq j \leq n),
$$

where $\omega_{n}$ is an $d^{n}$ th root of unity.

Proof. We proceed the proof by induction on $n$.

The case of $n=1$ is proved in Theorem 2.1. Assume that the result is true for $n-1$ and consider the case of $n$. Put $F_{j}=P\left(f_{j}, f_{j+1}\right)$ and $G_{j}=P\left(g_{j}, g_{j+1}\right)$. Then we can simplify the identity (3.1) into the form

$$
P_{n-1}\left(G_{0}, \ldots, G_{n-1}\right)=h^{d^{n}} P_{n-1}\left(F_{0}, \ldots, F_{n-1}\right) .
$$

It follows from the assumption of induction that

$$
G_{j}=\omega_{n-1} h^{d} F_{j} \quad(0 \leq j \leq n-1)
$$

or

$$
P\left(g_{j}, g_{j+1}\right)=\omega_{n-1} h^{d} P\left(f_{j}, f_{j+1}\right)
$$


From the result of $n=1$, we get

$$
g_{j}=\tilde{\omega}_{j} h f_{j} \text { and } g_{j+1}=\tilde{\omega}_{j} h f_{j+1}
$$

where $\tilde{\omega}_{j}$ are $d$ th root of $\omega_{n-1}$. Because of $f_{j} \not \equiv 0$ and $g_{j} \not \equiv 0(0 \leq j \leq n)$, we obtain $\tilde{\omega}_{0}=\cdots=\tilde{\omega}_{n-1}$ and set $\omega_{n}=\tilde{\omega}_{j}(0 \leq j \leq n-1)$. This completes the proof.

Q.E.D.

\section{§4. Constantness of holomorphic mappings}

In this section we prove theorems which show constantness of holomorphic mappings.

LEMMA 4.1. Let $f$ and $g$ be entire functions at least one of which are not identially equal to zero. If

$$
P(f, g)=\alpha^{d}
$$

for some entire function $\alpha$, then $(f: g)$ is constant.

Proof. Consider the factorization $P\left(w_{0}, w_{1}\right)=\prod_{j=1}^{d}\left(w_{0}+a_{j} w_{1}\right) . \quad$ If $\alpha \equiv 0$, then $f+a_{j} g \equiv 0$ for some $j$. Hence $(f: g)=\left(-a_{j}: 1\right)$. Assume that $\alpha \neq \equiv 0$. Since $\sum_{J=1}^{d}(1-(1 / d))=d-1>2$, we see that $(f: g)$ is constant by Theorem A.

Q.E.D.

THEOREM 4.2. Let $f_{0}, f_{1}$ and $f_{2}$ be entire functions at least two of which are not identially equal to zero, and let $C$ be a nonzero constant. If

$$
P\left(f_{0}, f_{1}\right)=C P\left(f_{1}, f_{2}\right)
$$

then $\left(f_{0}: f_{1}: f_{2}\right)$ is constant.

Proof. Note that the constantness of $\left(f_{0}: f_{1}\right)$ and that of $\left(f_{1}: f_{2}\right)$ are equivalent by Lemma 4.1 , and in this case trivially $\left(f_{0}: f_{1}: f_{2}\right)$ is constant.

Assume that neither $\left(f_{0}: f_{1}\right)$ nor $\left(f_{1}: f_{2}\right)$ are constant. Then by Theorem 2.1 , there exists $d$ th root $C^{\prime}$ of $C$ such that $f_{0}=C^{\prime} f_{1}, f_{1}=C^{\prime} f_{2}$, which is a contradiction.

Q.E.D.

For more $f_{j}$ 's, we have

THEOREM 4.3. Let $n \geq 2$ be an integer and $f_{0}, \ldots, f_{n}$ entire functions. If at least two of $P\left(f_{0}, f_{1}\right), P\left(f_{1}, f_{2}\right), \ldots, P\left(f_{n-1}, f_{n}\right)$ are not identically equal to zero and $\left(P\left(f_{0}, f_{1}\right): P\left(f_{1}, f_{2}\right): \cdots: P\left(f_{n-1}, f_{n}\right)\right)$ is constant, then $\left(f_{0}: \cdots: f_{n}\right)$ is constant.

Proof. We proceed the proof by induction on $n$. The case of $n=2$ is proved by Theorem 4.2.

Assume that the result of $n-1$ has been proved and consider the case of $n$. 
(I) The case of $P\left(f_{n-1}, f_{n}\right) \equiv 0$. Since in this case there exist at least two $j(0 \leq j \leq n-2)$ such that $P\left(f_{j}, f_{j+1}\right) \not \equiv 0,\left(f_{0}: \cdots: f_{n-1}\right)$ is constant by the assumption of induction. Further, the assumption $P\left(f_{n-1}, f_{n}\right) \equiv 0$ implies that $\left(f_{n-1}: f_{n}\right)=$ $\left(c_{n-1}: c_{n}\right) \neq(0: 1)$ or $f_{n-1}=f_{n} \equiv 0$. We can easily get the result by these.

(II) The case where $P\left(f_{n-1}, f_{n}\right) \not \equiv 0$ and there exist at least two $j(0 \leq j \leq n-2)$ such that $P\left(f_{j}, f_{j+1}\right) \not \equiv 0$. Then $\left(f_{0}: \cdots: f_{n-1}\right)=\left(c_{0}: \cdots: c_{n-1}\right)$ by the assumption of induction. If $P\left(f_{j}, f_{j+1}\right) \not \equiv 0$, then there exists a nonzero constant $C$ such that $P\left(f_{j}, f_{j+1}\right)=C P\left(f_{n-1}, f_{n}\right)$ by the constantness of $\left(P\left(f_{j}, f_{j+1}\right)\right.$ : $\left.P\left(f_{n-1}, f_{n}\right)\right)$. Because of $\left(f_{j}, f_{j+1}\right) \not \equiv(0,0)$ and $\left(f_{j}: f_{j+1}\right)=\left(c_{j}: c_{j+1}\right),\left(f_{n-1}: f_{n}\right)$ is constant by Lemma 4.1. If $f_{n-1} \not \equiv 0$, then we can write $f_{n}=\left(c_{n} / c_{n-1}\right) f_{n-1}$. If $f_{n-1} \equiv 0$, then we can write $f_{n}=\left(c_{n} / c_{j}\right) f_{j}$ or $f_{n}=\left(c_{n} / c_{j+1}\right) f_{j+1}$.

(III) The case where $P\left(f_{n-1}, f_{n}\right) \not \equiv 0$ and there exists the only one $j(0 \leq j \leq n-2)$ such that $P\left(f_{j}, f_{j+1}\right) \not \equiv 0$. If $P\left(f_{0}, f_{1}\right) \equiv 0$, we can get the result as the case $(\mathrm{I})$.

Hence we consider the case where $P\left(f_{0}, f_{1}\right) \not \equiv 0$ and $P\left(f_{1}, f_{2}\right)=\cdots=$ $P\left(f_{n-2}, f_{n-1}\right) \equiv 0$. In this case, we get $\left(f_{1}: \cdots: f_{n-1}\right)=\left(c_{1}: \cdots: c_{n-1}\right)$, where $c_{j}$ are nonzero constants, or $f_{1}=\cdots=f_{n-1} \equiv 0$. Moreover, we have $P\left(f_{0}, f_{1}\right)=$ $C P\left(f_{n-1}, f_{n}\right)$ for a nonzero constant $C$. If $f_{1}=\cdots=f_{n-1} \equiv 0$, then $f_{0}{ }^{d}=C f_{n}{ }^{d}$. Hence we get $\left(f_{0}: \cdots: f_{n}\right)=\left(c_{0}: 0: \cdots: 0: c_{n}\right)$. Consider the case of $\left(f_{1}: \cdots: f_{n-1}\right)=\left(c_{1}: \cdots: c_{n-1}\right)$. If $\left(f_{0}: f_{1}\right)$ is constant, then $\left(f_{n-1}: f_{n}\right)$ is constant and also we can write $f_{0}=\left(c_{0} / c_{1}\right) f_{1}, f_{n}=\left(c_{n} / c_{n-1}\right) f_{n-1}$, which implies $\left(f_{0}: \cdots: f_{n}\right)=\left(c_{0}: \cdots: c_{n}\right)$. If $\left(f_{0}: f_{1}\right)$ is not constant, then by Theorem 2.1, there exists a $d$ th root $C^{\prime}$ of $C$ such that $f_{0}=C^{\prime} f_{n-1}, f_{1}=C^{\prime} f_{n}$. Hence $\left(f_{0}: \cdots: f_{n}\right)$ is constant.

Q.E.D.

THEOREM 4.4. Let $n$ be a positive integer and $f$ a holomorphic mapping of $C$ into $\boldsymbol{P}^{n}(\boldsymbol{C})$ with a reduced representation $\left(f_{0}, \ldots, f_{n}\right)$. If $\boldsymbol{P}_{n}\left(f_{0}, \ldots, f_{n}\right) \equiv 0$, then $f$ is constant.

Proof. We proceed the proof by induction on $n$. The case of $n=1$ is trivial and the case of $n=2$ is proved easily by Theorem 4.2. Assume that the result for $n-1$ is proved. Let $P_{n}\left(f_{0}, \ldots, f_{n}\right) \equiv 0$. If we put $F_{j}=P\left(f_{j}, f_{j+1}\right)$, then $P_{n-1}\left(F_{0}, \ldots, F_{n-1}\right) \equiv 0$. If $F_{0}=\cdots=F_{n-1} \equiv 0$, then the conclusion is obvious. Hence assume that there exists $j(0 \leq j \leq n-1)$ such that $F_{j} \not \equiv 0$. Note that there exist at least two such $j$. By the assumption of induction, $\left(F_{0}: \cdots: F_{n-1}\right)$ is constant. Using Theorem 4.3 , we conclude that $\left(f_{0}: \cdots: f_{n}\right)$ is constant.

Q.E.D.

It follows from this theorem and Brody's theorem (see Theorem 2.1 in [L, p. 68]) that the hypersurface $S_{n}$ defined by $P_{n}\left(w_{0}, \ldots, w_{n}\right)=0$ in $\boldsymbol{P}^{n}(\boldsymbol{C})$ is Kobayashi-hyperbolic.

THEOREM 4.5. Let $f$ be a holomorphic mapping of $\boldsymbol{C}$ into $\boldsymbol{P}^{2}(\boldsymbol{C})$ with a reduced representation $\left(f_{0}, f_{1}, f_{2}\right)$. If $P_{2}\left(f_{0}, f_{1}, f_{2}\right)=\alpha^{d^{2}}$ for some entire function $\alpha \neq 0$, then $f$ is constant. 
Proof. Put $F_{0}=P\left(f_{0}, f_{1}\right)$ and $F_{1}=P\left(f_{1}, f_{2}\right)$. Then $P\left(F_{0}, F_{1}\right)=\alpha^{d^{2}}$. Trivially $\left(F_{0}, F_{1}\right) \not \equiv(0,0)$. In the case of $F_{0} \equiv 0$, we have $F_{1}{ }^{d}=\alpha^{d^{2}}$. It follows from $F_{0} \equiv 0$ that $f_{0}=f_{1} \equiv 0$ or that $\left(f_{0}: f_{1}\right)=\left(c_{0}: c_{1}\right) \neq(1: 0),(0: 1)$. By Lemma 4.1 , it follows from $F_{1}^{d}=\alpha^{d^{2}}$ that $\left(f_{1}: f_{2}\right)=\left(c_{1}^{\prime}: c_{2}^{\prime}\right)$. If $f_{0}=f_{1} \equiv 0$, then $f_{2}=\omega_{2} \alpha$, where $\omega_{2}$ is a $d^{2}$ th root of unity. Hence $\left(f_{0}: f_{1}: f_{2}\right)=(0: 0: 1)$. If $\left(f_{0}: f_{1}\right)=\left(c_{0}: c_{1}\right)$, then $c_{1} \neq 0$, which implies the constantness of $\left(f_{0}: f_{1}: f_{2}\right)$. In the case of $F_{1} \equiv 0$, we have $F_{0}{ }^{d}=\alpha^{d^{2}}$. Hence we can prove our assertion as above. If $F_{0} \not \equiv 0$ and $F_{1} \equiv 0$, then $\left(F_{0}: F_{1}\right)$ is constant by Lemma 4.1. In this case we can use Theorem 4.2 and get the conclusion.

Q.E.D.

THEOREM 4.6. Let $f$ be a holomorphic mapping of $\boldsymbol{C}$ into $\boldsymbol{P}^{n}(\boldsymbol{C})$ with a reduced representation $\left(f_{0}, \ldots, f_{n}\right)$. If $P_{n}\left(f_{0}, \ldots, f_{n}\right)=\alpha^{d^{n}}$ for some entire function $\alpha \not \equiv 0$, then $f$ is constant.

Proof. We proceed the proof by induction on $n$. The case of $n=1$ is proved by Lemma 4.1 and the case of $n=2$ by Theorem 4.5. Assume that the result for $n-1$ is proved. Let $P_{n}\left(f_{0}, \ldots, f_{n}\right)=\alpha^{d^{n}}$. If we put $F_{j}=P\left(f_{j}, f_{j+1}\right)$, then $P_{n-1}\left(F_{0}, \ldots, F_{n-1}\right)=\alpha^{d^{n}}$. Since the case $F_{0}=\cdots=F_{n-1} \equiv 0$ is impossible, there exists $j(0 \leq j \leq n-1)$ such that $F_{j} \not \equiv 0$. By the assumption of induction, $\left(F_{0}: \cdots: F_{n-1}\right)$ is constant. If there exist at least two $j(0 \leq j \leq n-1)$ such that $F_{j} \not \equiv 0$, then Theorem 4.3 yields the conclusion. Hence we consider the case where there exists the only one such $j$. If $F_{j} \not \equiv 0$, then $K F_{j}^{d^{n-1}}=\alpha^{d^{n}}$, where $K$ is a nonzero constant. Hence $K^{\prime} F_{j}=\alpha^{d}$, where $K^{\prime}$ is an $d^{n-1}$ th root of $K$. Therefore $\left(f_{j}: f_{j+1}\right)$ is constant by Lemma 4.1 . In this case, if $f_{j} \equiv 0$, then $f_{0}=\cdots=f_{j} \equiv 0$ and $\left(f_{j+1}: \cdots: f_{n}\right)=\left(c_{j+1}: \cdots: c_{n}\right)$, where $c_{k}$ are nonzero constants. If $f_{j+1} \equiv 0$, then $f_{j+1}=\cdots=f_{n} \equiv 0$ and $\left(f_{0}: \cdots: f_{j}\right)=\left(c_{0}: \cdots: c_{j}\right)$, where $c_{k}$ are nonzero constants. In the case where $f_{j} \not \equiv 0$ and $f_{j+1} \not \equiv 0$, we have the conclusion by using $F_{k} \equiv 0(k \neq j)$.

Q.E.D.

From this theorem, every holomorphic mapping of $C$ into $P^{n}(C)$ omitting $S_{n}$ is constant. Hence $\boldsymbol{P}^{n}(\boldsymbol{C}) \backslash S_{n}$ is hyperbolically imbedded in $\boldsymbol{P}^{n}(\boldsymbol{C})$ and is complete hyperbolic by Theorem 3.3 in [L, p. 75].

\section{REFERENCES}

[L] S. LANG, Introduction to Complex Hyperbolic Spaces, Springer-Verlag, New York-BerlinHeidelberg, 1987.

[S] M. SHIRosakI, On polynomials which determine holomorphic mappings, J. Math. Soc. Japan, 49 (1997), 289-298.

Department of Mathematical Sciences

COLlEGE OF ENGINEERING

OSAKa PREFECTURe UNIVERSITY

599-8531 SAKAI, JAPAN 